\title{
A new threat to India- Zika! An obstetrician's perspective
}

\author{
Renuka Malik ${ }^{1 *}$, Nidhi Garg1, Kaveri Khera² \\ ${ }^{1}$ Department of Obstetrics and Gynecology, PGIMER and DR RML Hospital, New Delhi, India
}

${ }^{2}$ Microbiologist, Santa Barbara, California, USA

Received: 06 August 2017

Accepted: 04 September 2017

\section{*Correspondence:}

Dr. Renuka Malik,

E-mail: renucam@yahoo.co.in

Copyright: (C) the author(s), publisher and licensee Medip Academy. This is an open-access article distributed under the terms of the Creative Commons Attribution Non-Commercial License, which permits unrestricted non-commercial use, distribution, and reproduction in any medium, provided the original work is properly cited.

\begin{abstract}
Since last 2 years, ever since the Zika outbreak in Brazil, there has been a great interest in this infection caused by Aedes aegypti and A. albopictus, though this RNA Flavirus has been in existence since 1947. A large number of children born with microcephly during the outbreak in Brazil attributed to Zika was alarming. Though Brazil in May, 1917 has declared an end to Zika emergency, this virus continues to be detected in parts of America ca and south East Asia, including Thailand, Cambodia, Malaysia and Myanmar. WHO declared Zika as Public Health Emergency of International Concern on $1^{\text {st }}$ February, 2016. Though at present only 4 confirmed cases have been reported in India, Zika is a notifiable disease along with chicken guinea and dengue. A state of awareness and preparedness is required, among treating physicians, obstetricians and pediatricians for this disease. This article deals with history, presentation, diagnosis, pitfalls, management and preventive aspect of Zika in pregnancy.
\end{abstract}

Keywords: Aedes aegypti, Fever, Microcephly, Rash, Zika

\section{INTRODUCTION}

Since 2015, Zika virus has become a new global issue because of its unique property to reside in body fluids for long and neuropathic teratogenicity. ${ }^{1}$ However, the existence of Zika virus and its vector are not new.

\section{Epidimology}

Zika virus is a flavivirus which was first isolated in 1947 from monkey in the Zika Forest of Uganda and later in 1954; the first 3 cases of human infection were reported in Nigeria. It is spread by daytime-active Aedes mosquitoes, such as A. aegypti and A. albopictus. Serosurveillance studies in humans suggest that Zika virus is widespread throughout Africa, Asia, and Oceania and until now, sporadic bursts have been noted. This pattern changed in 2007, when the first major outbreak of
Zika virus infection occurred in Yap (Federated States of Micronesia), where $73 \%$ of the population was infected and symptomatic disease developed in $18 \%$ of infected persons. $^{1-3}$ A further outbreak occurred in French Polynesia in 2013. Outbreak in Easter Island in 2014 marked the first arrival of Zika virus in the Americas. This was followed in May 2015 by confirmation of cases in northeast Brazil, where again the Zika virus sequence belonging to the Asian lineage was found. Brazil has reported more than 4000 cases of suspected microcephaly that may be linked to Zika. Colombia reported local transmission in October 2015. India has reported 4 cases as of now with no reported case of microcephaly or Gullian barre syndrome. ${ }^{3}$

However, these studies may overestimate the virus's true prevalence, given serologic overlap between Zika virus and related flaviviruses such as dengue virus. Between $1^{\text {st }}$ January 2007 and $3^{\text {rd }}$ March 2016, a total of 52 countries 
and territories have reported autochthonous (local) transmission or indication of transmission of Zika virus. Sexual transmission of virus is also reported. History of travel to endemic areas and blood transfusion is also implicated. However, the recent emergence and spread of ZIKV in the Americas has resulted in the WHO declaration of Public Health Emergency of International Concern, on $1^{\text {st }}$ February, 2016 due to the potential association between the infection and prenatal microcephaly or other brain anomalies. ${ }^{3,5}$ Recent studies have suggested ZIKV infections to be highly neurotrophic, with a few cases reporting association with bilateral macular and perimacular lesions in eye. Guillain-Barré syndrome (GBS) was associated with the Zika virus outbreaks in Brazil and Venezuela., Presumptive sexual transmission has been reported in two cases. Isolation of virus in semen supports potential sexual transmission. Zika virus was detected in approximately $3 \%$ of asymptomatic blood donors during the French Polynesian outbreak, suggesting that transmission might be possible through infected blood and blood products. Evidence implies transplacental transmission and perinatal transmission during delivery, with Zika virus RNA being found in amniotic fluid and in paired blood samples taken from newborn infants and mother.,

\section{Presentation, diagnosis and management}

From the obstetrician view point any maculo-papular rash with fever $>37.2^{\circ} \mathrm{C}$ occurring in pregnancy with conjuctivitis, myalgia, headache, pruritus, malaise or arthalgia should be thought as viral infection in endemic regions. Adverse outcomes have been noted regardless of the trimester during which the women were infected with ZIKV $(55 \%$ of pregnancies had adverse outcomes after maternal infection in the first trimester, 52\% after infection in the second trimester, and $29 \%$ after infection in third trimester. ${ }^{6}$ Infection occurring in pregnancy in endemic area or history of travel to endemic areas within two weeks is suspicious of Zika. Primary infection is mild lasting 2-7 days but maybe asymptomatic in $2 / 5$ of patients. Symptomatic treatment is given in the form of antipyretics and analgesics. There is no specific antiviral treatment for Zika. Supportive nursing care and relief of symptoms are the standard treatment.

Use paracetamol for fever. Aspirin is to be avoided. There is no specific treatment for Zika. At present here is no vaccine available for Zika The differential diagnosis of Zika virus infection is wide. Diagnosis is guided by history (countries of travel, sexual contacts, and contact with other cases of infection) and examination. Samples like blood, urine, placenta, and amniotic fluid sent for triple testing for dengue, chikungunya and Zika. Lab confirmation is by testing sample for antibodies Elisa IgM and then confirming it by RT-PCR. ${ }^{1,8}$ Zika virus has been also isolated from breast milk. In addition to dengue and chikungunya, other diagnoses that should be considered include HIV seroconversion, measles, scarlet fever, rickettsial infection, leptospirosis, parvovirus, enterovirus, rubella, and secondary syphilis. The symptoms and clinical signs do not have sufficient positive or negative predictive value and therefore laboratory testing is needed for reliable diagnosis. ${ }^{7,8}$ Arthalgias are more commonly seen in chicken guinea and thrombocytopenia is a feature of dengue. RCOG, in its interim guidelines published just recently in August has made an algorithm for pregnant patients travelling to endemic areas which consists of getting fetal ultrasound at 18-20 weeks and if normal repeating at 28-30 weeks. ${ }^{9}$

Zika viral infection can result in abortion, IUD or child born with congenital Zika. ZIKV infection included a broad range of fetal and newborn neurological and ocular abnormalities; fetal growth restriction, stillbirth, and perinatal death. Microcephaly was the primary neurological complication reported in eight studies, with an incidence of about $1 \%$ among newborns of ZIKV infected women in one study. ${ }^{6}$ Congenital Zika acquired through transplacental route there are brain abnormalities with and without microcephaly, intracranial calcifications, cerebral atrophy, abnormal cortical formation (polymicrogyria, lissencephaly, pachygyria, schizencephaly), gray matter heterotopia,corpus callosum abnormalities cerebellar abnormalities, ventriculomegaly, hydrocephaly, neural tube defects (anencephaly, acrania, encephalocele, spina bifida, holoprosencephaly and structural eye abnormalities like microphthalmia, anophthalmia, coloboma, cataract, intraocular calcifications, chorioretinal anomalies involving the macula, mottling and retinal hemorrhage), optic nerve atrophy have been documented. congenital contractures (e.g., arthrogryposis, club foot, congenital hip dysplasia) with associated brain abnormalities, congenital deafness documented by postnatal test. ${ }^{7}$ In Live births measured head circumference (HC) adjusted for gestational age and sex <3rd percentile at birth, or if not measured at birth, within first 2 weeks of life; For microcephaly the standard used in US is prenatal HC more than 3 standard deviations below the mean based on ultrasound or postnatal $\mathrm{HC}<3 \mathrm{rd}$ percentile below gestational age specific measurements is important though it occurs in $1 \%-13 \%$ of patients. ${ }^{8}$

Laboratory evidence of a confirmed recent Zika virus infection includes detection of Zika virus or Zika virus RNA or antigen in any body fluid or tissue specimen or antibody detection. Several methods can be used for diagnosis, such as viral nucleic acid detection, virus isolation and serological testing. Diagnosis by serology can be difficult as the virus can cross react with other flaviviruses. Nucleic acid detection by reverse transcriptase-polymerase chain reaction (RT-PCR) targeting the non-structural protein 5 genomic region is the primary means of diagnosis. Standard RT-PCR and quantitative RT-PCR provide a rapid, specific and sensitive method for ZIKV early detection. Viral RNA has been detected in serum up to day 10 after the onset of symptoms. Serology testing with positive or equivocal 
Zika virus or dengue virus IgM test on serum with a positive titer for Zika virus $(\geq 10)$ from plaque reduction neutralization testing (PRNT) together with negative PRNT titer (i.e., <10) for dengue virus, is confirmatory. ${ }^{9}$ Concurrent RNA nucleic acid test (NAT) testing and Zika virus IgM testing is recommended as soon as possible or through 12 weeks after symptom onset. PRNT is a confirmatory diagnosis. ${ }^{10}$ As disease manifestations of dengue and chikungunya virus infections can resemble those of Zika virus infection, additional testing for these viruses are considered to aid in differentiating dengue and chikungunya virus infections. As of May 3, 2017, serum is the primary diagnostic specimen for Zika virus.

The Zika MAC-ELISA can also be used to test CSF when collected alongside a patient-matched serum. Positive and equivocal Zika MAC-ELISA results are not definitive for diagnosis of Zika virus infection. False positive results may occur in some patients with recent, closely-related flavivirus infections, such as dengue infections. In patients who have received yellow fever or Japanese encephalitis vaccination, cross-reactive antibodies in both the IgM and neutralizing antibody assays may make it difficult to identify which flavivirus is causing the patient's current illness. Absence of laboratory evidence of Zika virus infection cannot definitively rule out Zika virus infection in persons with epidemiological risk factors. All results should be considered in the context of clinical signs and symptoms, exposure risk and time since symptom onset or in the absence of symptoms on time since exposure.

\section{Global measures}

The global prevention and control strategy launched by WHO as a Strategic Response Framework encompasses surveillance, response activities and research. WHO has divided the countries into four categories, with India in fourth category. It has confirmed 4 confirmed case of Zika in India, category not yet revised.

- Category 1: Area with new introduction or reintroduction with ongoing transmission including United States of America;

- Category 2: Area either with evidence of virus circulation before 2015 or area with ongoing transmission that is no longer in the new or reintroduction phase, but where there is no evidence of interruption which includes African Republic, Indonesia, Thailand, Bangladesh, Malaysia, Philippines, Viet Nam;

- Category 3: Area with interrupted transmission and with potential for future transmission French Polynesia;

- Category 4: Area with established competent vector but no known documented past or current transmission, which includes India, Bhutan and Sri Lanka.
Malaysia is one country which gives Zika free blood tranfusion, if required in pregnant women.UK does not accept blood donation if the donor has travelled to endemic areas in preceding 6 months. RCOG has introduced its guidelines for Zika in pregnancy, which includes laboratory testing and ultrasound for fetal anamolies revised recently on $2^{\text {nd }}$ August $2017 .{ }^{9}$

Brazil launched a major mosquito eradication program last year and in May 2017 has announced the end of its public health emergency over Zika, the mosquito-borne virus that became a global concern just 18 months ago. Brazil's eradication program involved thousands of health workers who fumigated about 20 million homes. The country also experimented with other methods, including the release of genetically modified mosquitoes which were so altered so they when they mated with other $A$. aegypti their offspring were born sterile, preventing a new generation. Bacterium Wolbachia was introduced which when after infecting $A$. aegypti prevents the mosquitoes from transmitting the disease. ${ }^{1,3}$

For the first time Zika vaccine is being tested in pregnant volunteers, in phase II trials. A controversy erupted when none of pregnant women in Combodia having confirmed Zika infection showed no incidence of microceply. In June,2016 the New England Complex System Institute released a report doubting Zika as cause of microcephly.A group of Argentine doctors suggested the larvicidal pesticide pyriproxyfen might actually be causing the microcephaly epidemic, this has been refuted by $\mathrm{WHO}$ as there is no evidence for it. ${ }^{3}$

\section{Measures in India}

India is a high risk zone because of presence of competent vector Aedes. The low incidence may be because of some cross acquired immunity, which is unclear at present. Zika, chikungunya and dengue are notifiable disease in India. On 15 May 2017, the Ministry of Health and Family Welfare (MoHFW) reported three laboratory confirmed cases of Zika virus disease in Bapunagar area, Ahmadabad, Gujarat- one antenatal, one postnatal and one male with fever. No case of congenital Zika syndrome has been reported in India. ${ }^{3}$ Fourth case has been recently reported from Tamil Nadu.

National guidelines and action plan on Zika virus disease have been shared with the states to prevent an outbreak of Zika. In addition to National Institute of Virology, Pune, and NCDC in Delhi, 25 laboratories have also been strengthened by Indian Council of Medical Research (ICMR) for laboratory diagnosis. In addition, 3 entomological laboratories are conducting Zika virus testing on mosquito samples. ICMR has tested many human samples and mosquito samples for the presence of Zika virus but found them negative for Zika. In India, 55 sentinel sites are monitoring microcephly Airports and seaports have been issued directives to be on guard. Rapid response teams have been created. ${ }^{3,12,14}$ 
As preventive measures, pregnant women should be told to use repellents, wear light colored, long sleeved clothes and ensuring rooms are fitted with screens to prevent mosquitoes from entering. N, N-diethyl meta toluamide (DEET) based repellents are the most effective insect repellents widely available, and have been in use for over 50 years. Preparations with concentrations of DEET up to $50 \%$ are commonly available and are safe in pregnant and breastfeeding women and in infants and children over the age of 2 months. ${ }^{13}$ Larvicidal drugs should be added to large water containers like coolers. In addition because of the risk of sexual transmission, it is critical for men and women to receive counseling on travel and safer sexual practices and be offered condom. Vaccine against Zika is being developed and many vaccines have entered phase II trial. Phase I trials of this vaccine have been very promising. Whether this vaccine can be given in pregnancy remains to be seen. At present, testing for Zika in blood donors is not done in India.

Zika virus at present remains a potential threat in India, because of presence of competent vector Aedes and occurrence in neighboring countries like Malaysia, Thailand, Philippines and Cambodia. Though at present there is no reason for alarm but awareness and preparedness of physicians, obstetricians and pediatricians is required. MOHFW Website provides information on Zika, including a travel advisory. ${ }^{14}$

Funding: No funding sources Conflict of interest: None declared

Ethical approval: Not required

\section{REFERENCES}

1. Zika virus/CDC. 2017. Available at http://www.cdc. gov/zika/index.html. Accessed on 21 July 2017.

2. Plourde AR, Bloch EM. A Literature Review of Zika Virus. Emerging Infectious Dis. 2016;22(7):1185-92.

3. Zika virus/complications/WHO. 2016. Available at http://www.who.int/emergencies/zika-virus/media- centre/webcast-18-november-2016/en/. Accessed on 23 July 2017.

4. Campos GS, Bandeira AC, Sardi SI. Zika Virus Outbreak, Bahia, Brazil. Emerg Infect Dis. 2015;21(10):1885-6.

5. Ozkurt Z, Tanriverdi EC. Global Alert: Zika Virusan Emerging Arbovirus. Eurasian J Med. 2017;49(2):142-7.

6. Chibueze EC, Tirado V. Zika virus infection in pregnancy: a systematic review of disease, course and complications. Reprod Health. 2017;14:28.

7. Bell BP, Boyle CA, Petersen LR. Preventing Zika Virus Infections in Pregnant Women: An Urgent Public Health Priority. Am J Public Health. 2016;106(4):589-90.

8. Brasil P, Pereira JP, Moreira ME, Nogueira RMR, Damasceno L, Wakimoto M, et al. Ribeiro Nogueira, Zika Virus Infection in Pregnant Women in Rio de Janeiro. N Engl J Med. 2016;375:2321-34.

9. Musso D, Gubler DJ. Zika virus. Clin Microbiol Rev. 2016;29:487-524.

10. Sharma A, Lal SK. Zika Virus: Transmission, Detection, Control, and Prevention. Frontiers in Microbiology. 2017;8:110.

11. Zika virus (ZIKV): clinical and travel guidance. Available at: https://www.gov.uk/government/ collections/zika-virus-zikv-clinical-and-travelguidance. Accessed on 7 August 2017.

12. Basarab M, Bowman C, Aarons EJ, Cropley Ian, Zika Virus. Br Med J. 2016;352:i1049.

13. Insect and tick bite avoidance. Available at: http://travelhealthpro.org.uk/insect-tickbiteavoidance/. Accessed on 3 August 2017.

14. Available at: http://mohfw.nic.in/media/diseasealerts. Accessed on 3 August 2017.

Cite this article as: Malik R, Garg N, Khera K. A new threat to India- Zika! An obstetrician's perspective. Int J Reprod Contracept Obstet Gynecol 2017;6:4209-12. 